\title{
A Solid-Phase Synthesis of 5-Isoxazol-4-yl-[1,2,4]oxadiazoles
}

Chao Quan and Mark J. Kurth

\author{
Department of Chemistry \\ University of California \\ One Shields Avenue \\ Davis, CA 95616-5295
}

Supporting Information Available (20 pages):

General experimental information (page 2).

${ }^{1} \mathrm{H}$ NMR and ${ }^{13} \mathrm{C}$ NMR for compounds $3,10 \mathrm{~b}, 10 \mathrm{c}, 10 \mathrm{~h}, 10 \mathrm{i}, 10 \mathrm{~m}, 10 \mathrm{p}, 10 \mathrm{q}$, and 10r.

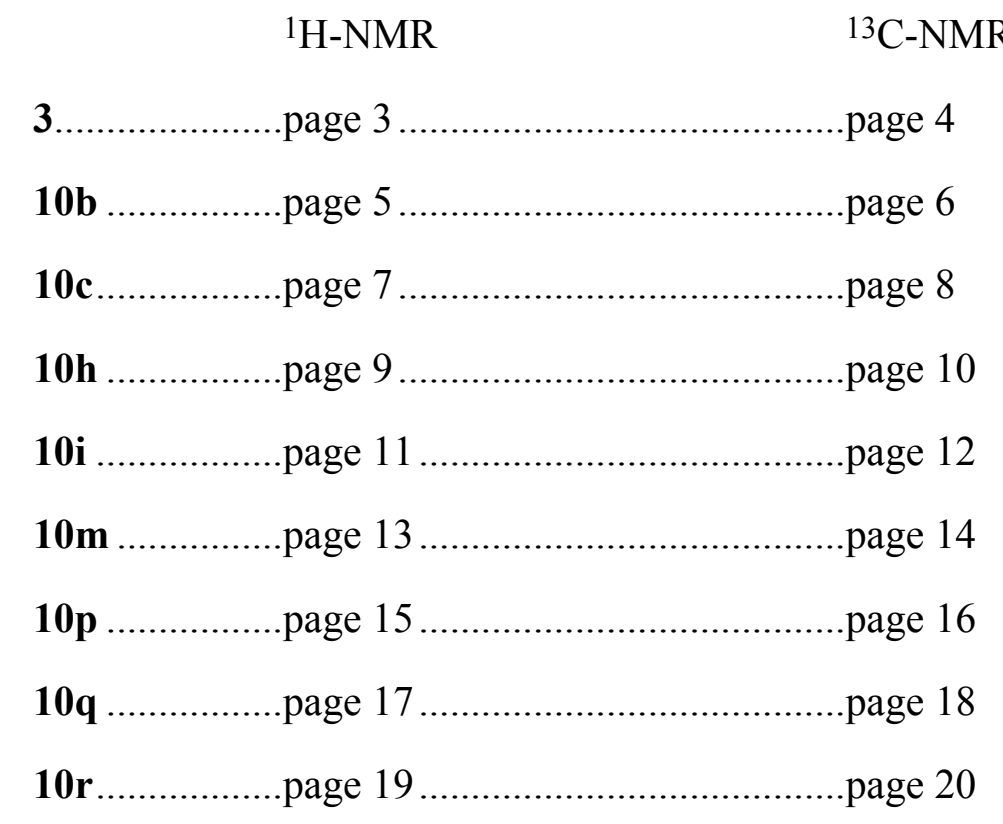


General Information. Anhydrous THF was distilled from sodium metal and methylene chloride was distilled from $\mathrm{CaH}_{2} .{ }^{1} \mathrm{H}$ NMR and ${ }^{13} \mathrm{C}$ NMR were recorded at ${ }^{1} \mathrm{H}$ at $400 \mathrm{MHz}$ and $100 \mathrm{MHz}$, respectively. $\mathrm{CDCl}_{3}$ was used as internal standard for ${ }^{13} \mathrm{C} \mathrm{NMR}$, and tetramethylsilane was added as internal standard for ${ }^{1} \mathrm{H}$ NMR. Infrared spectra were recorded neat. After reaction work-up, solutions were dried using $\mathrm{Na}_{2} \mathrm{SO}_{4}$ and solvents were subsequently removed by rotary evaporation. For solid-phase reactions, the resin was washed thoroughly with DCM (3x), MeOH (3x), and DCM $(3 x)$. 


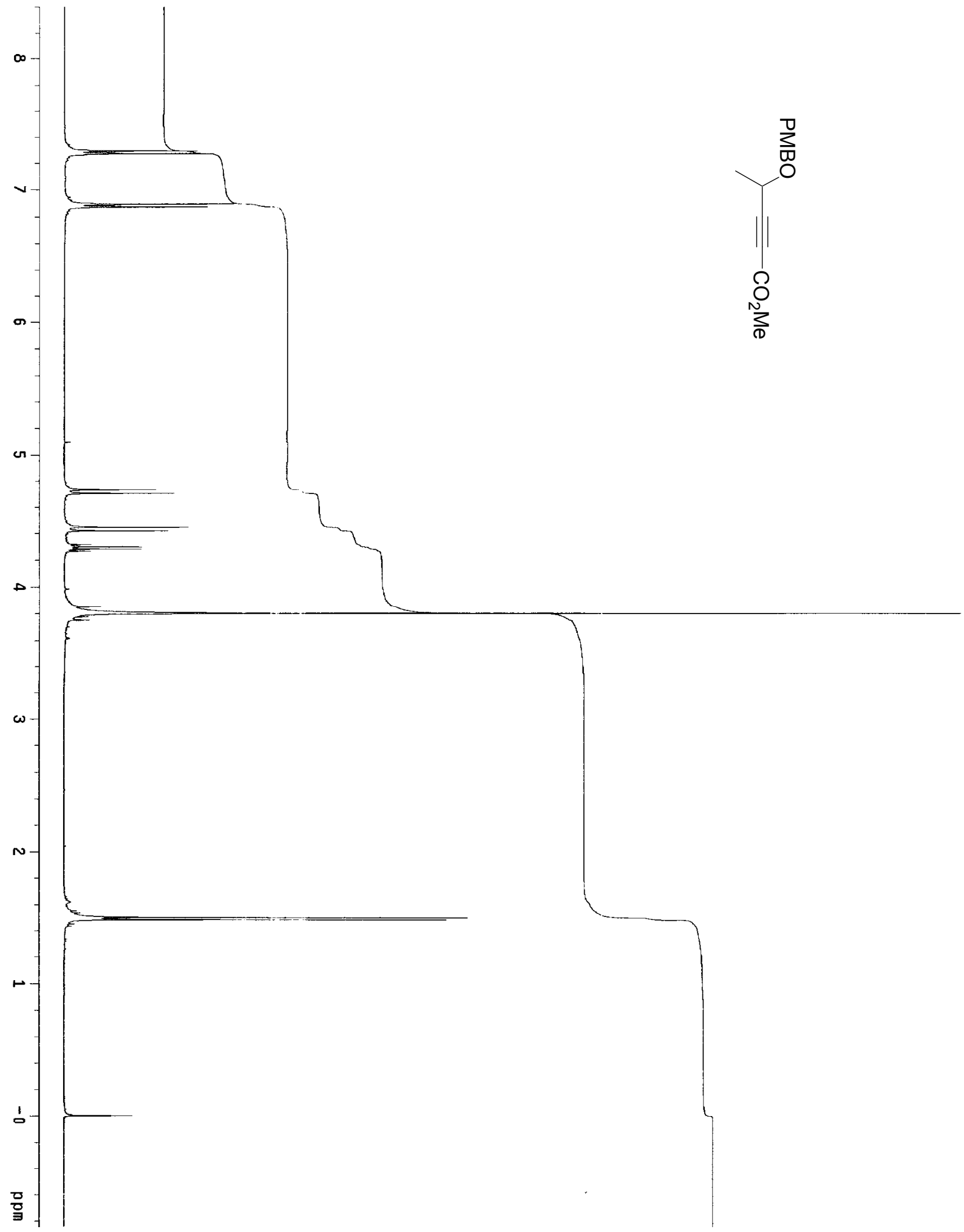



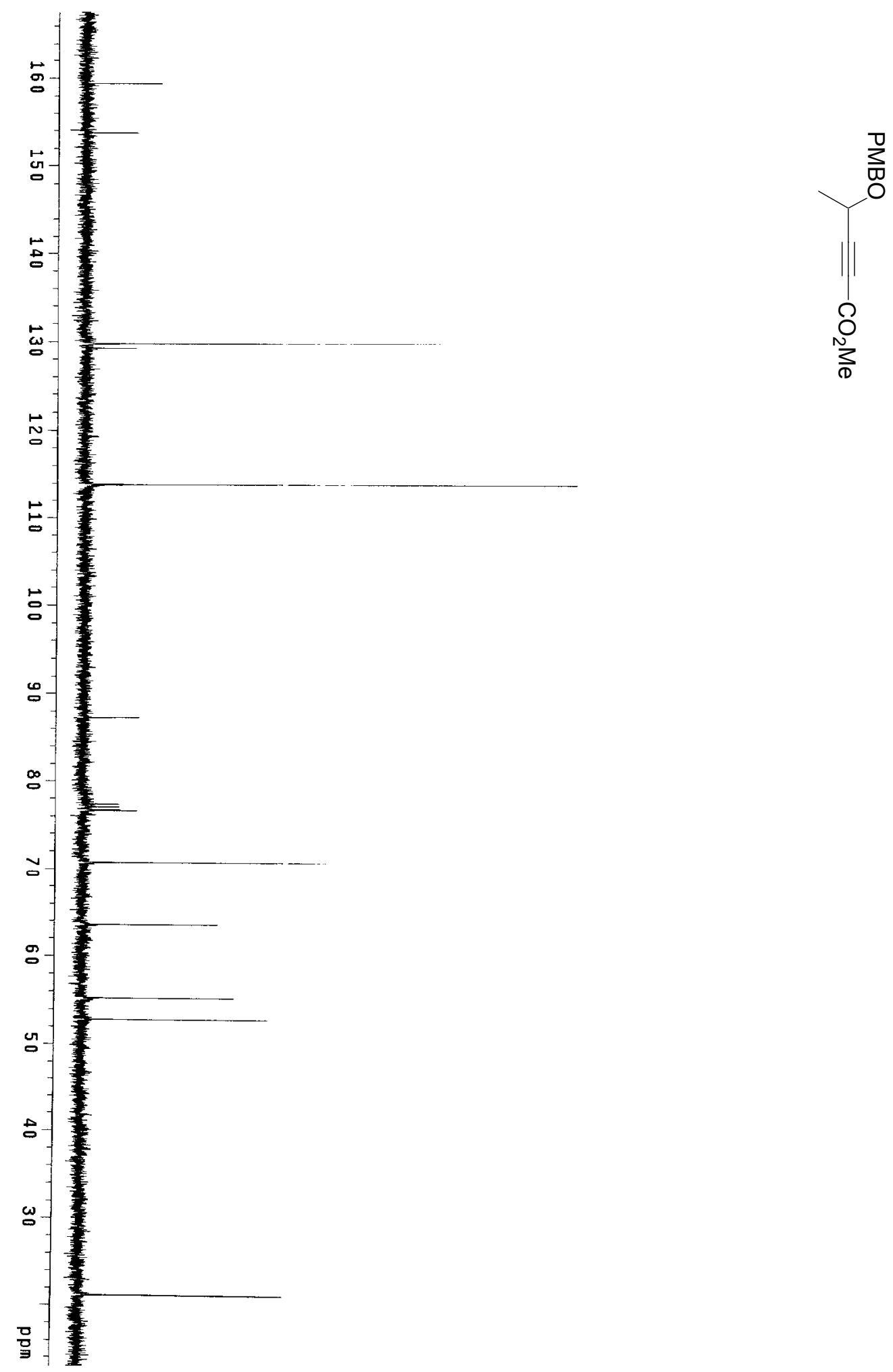


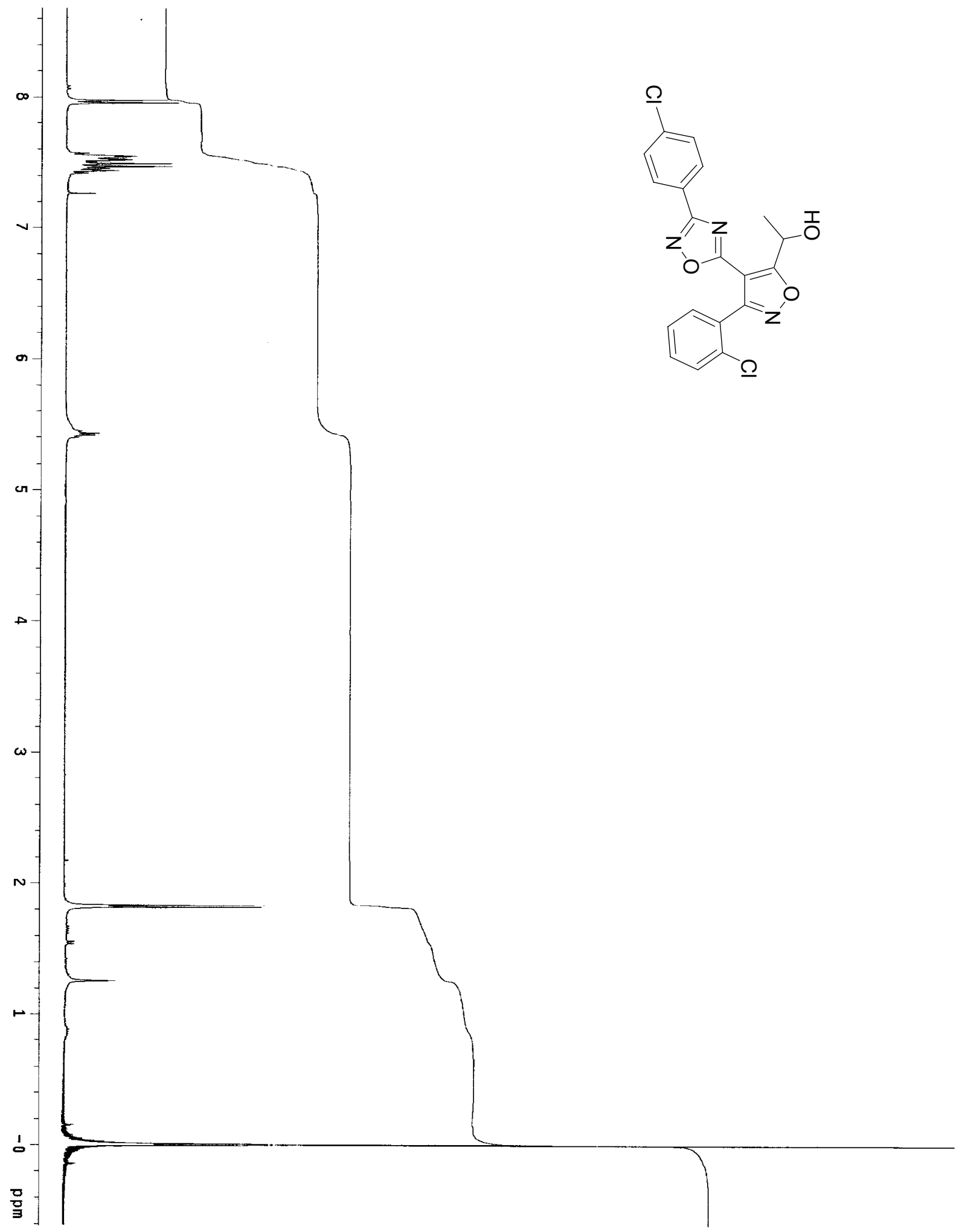



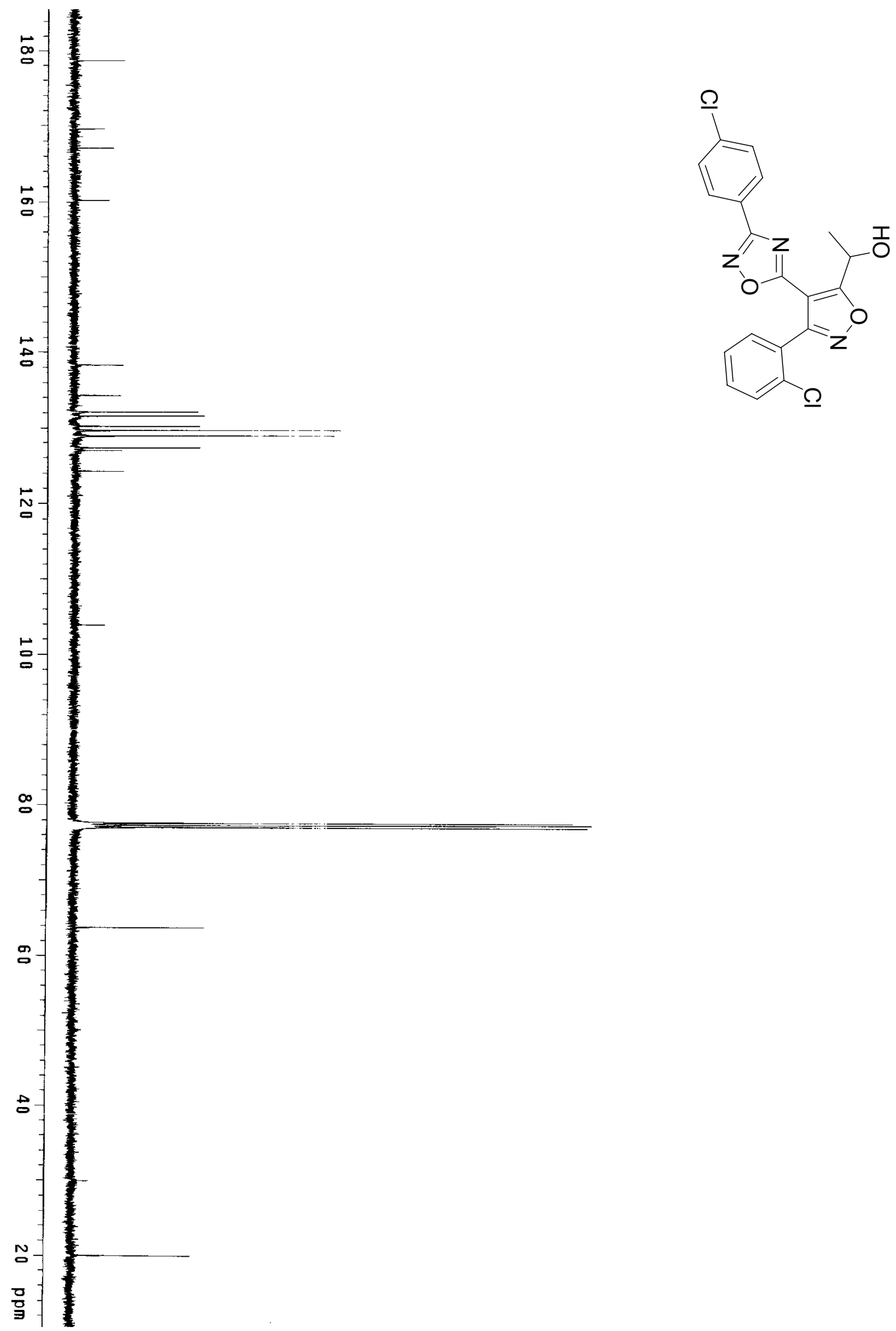


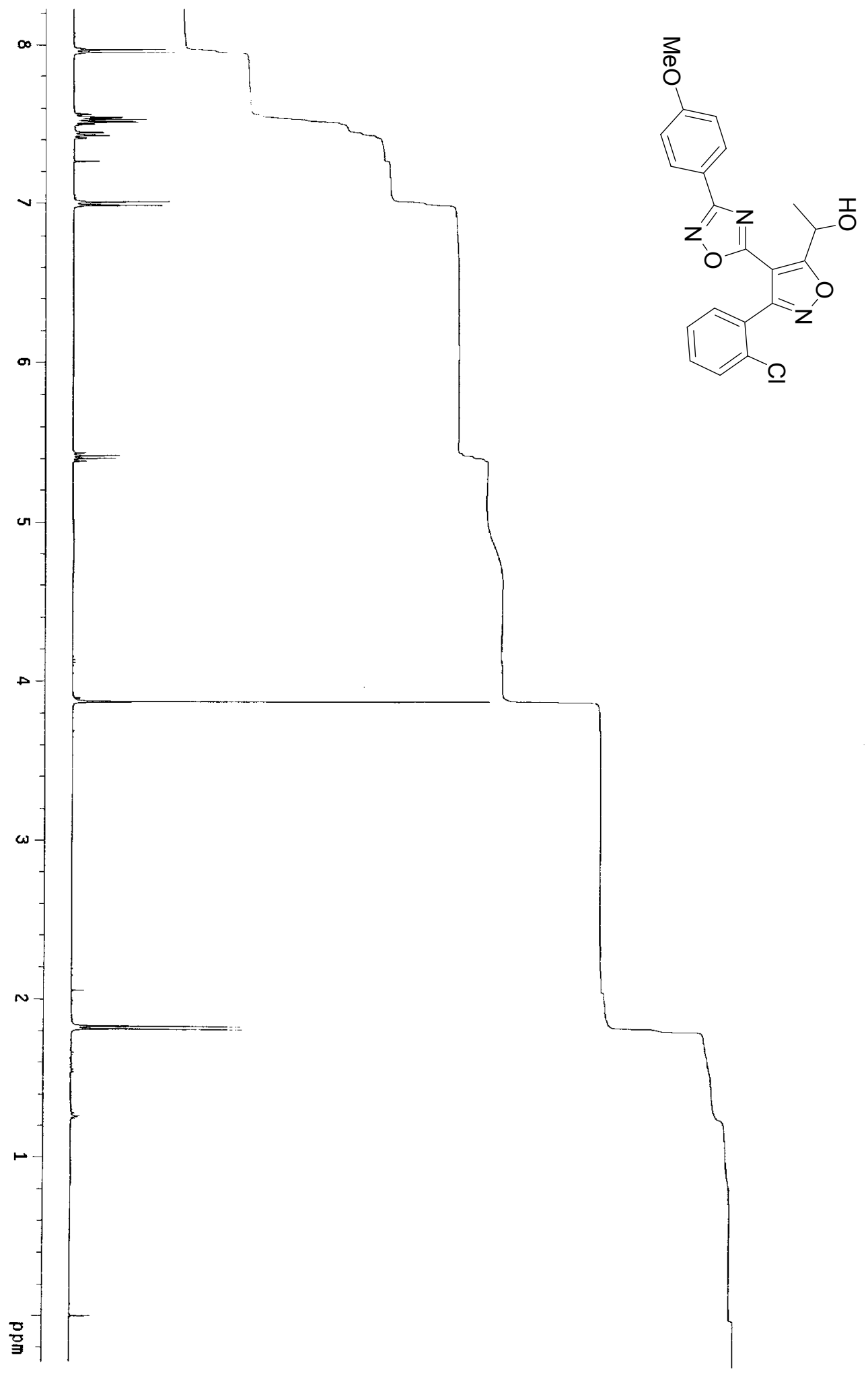



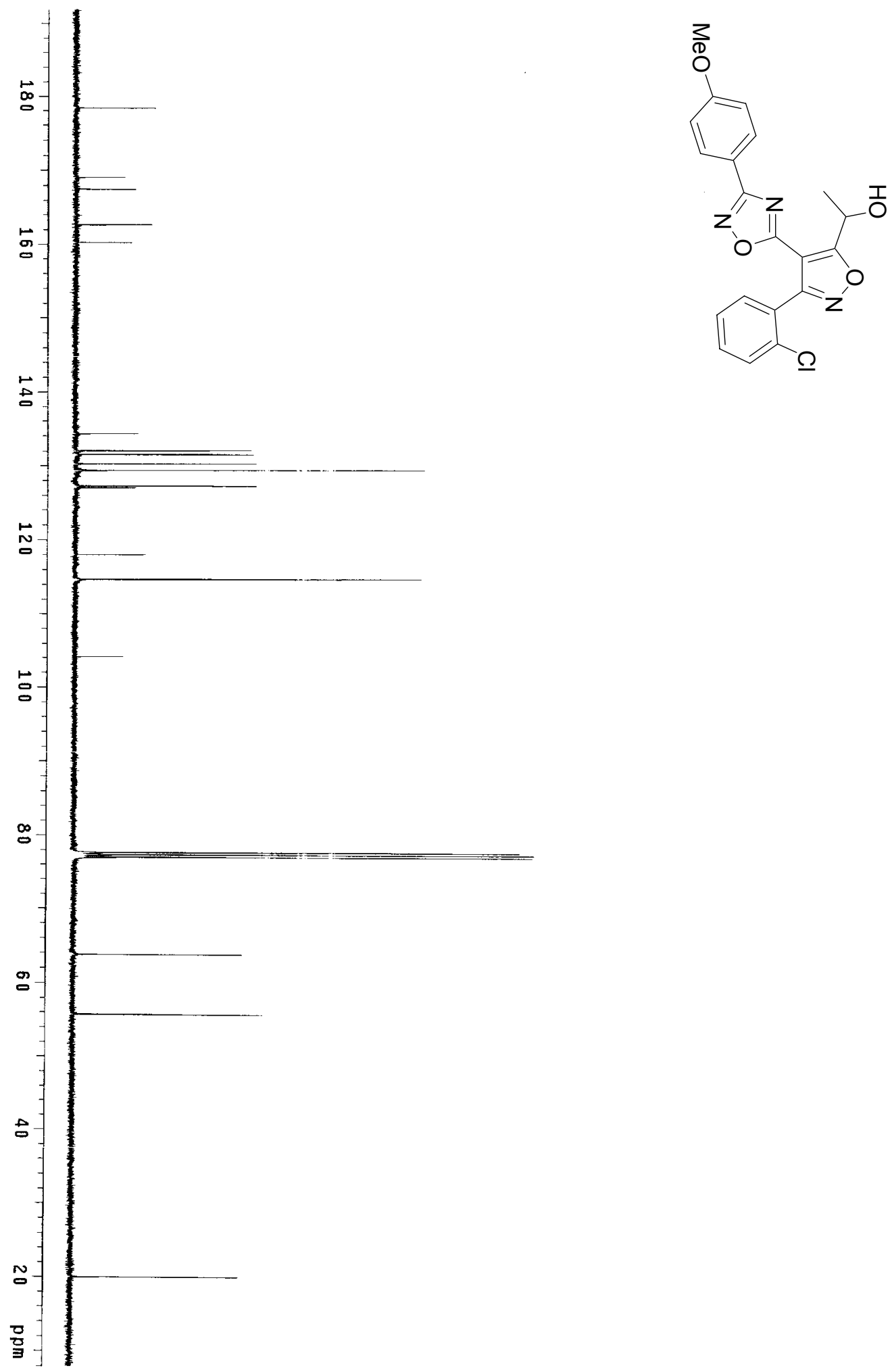


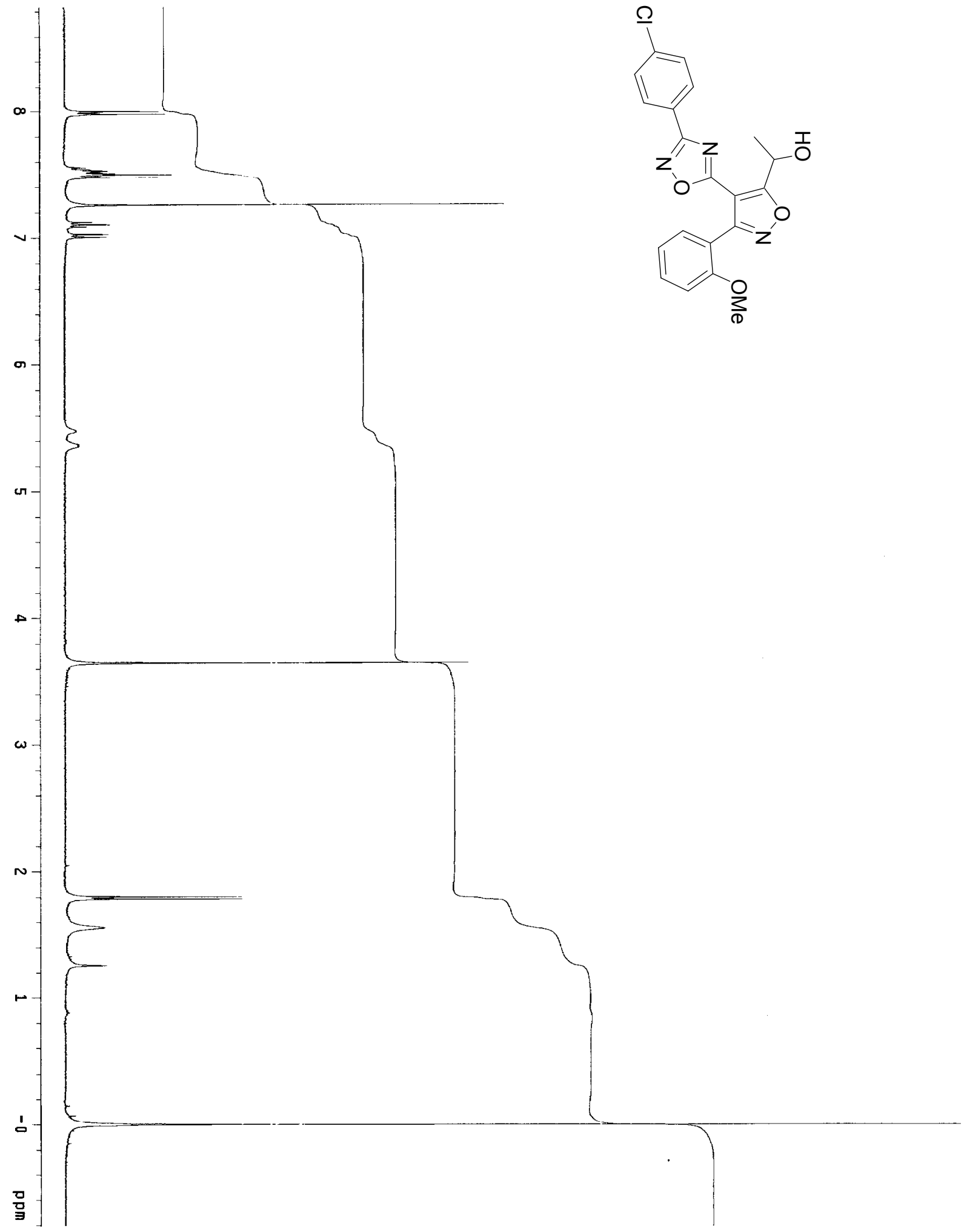



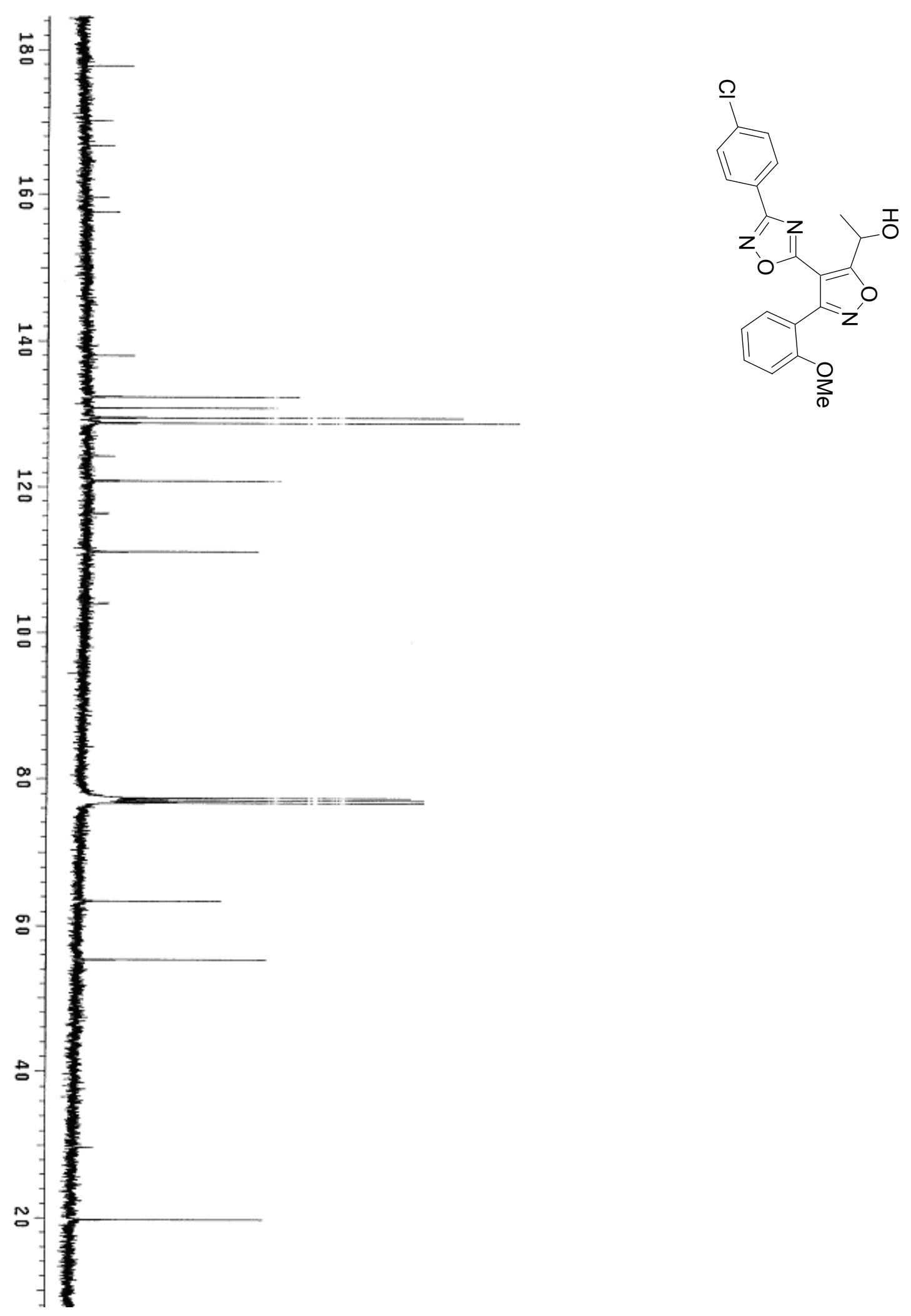


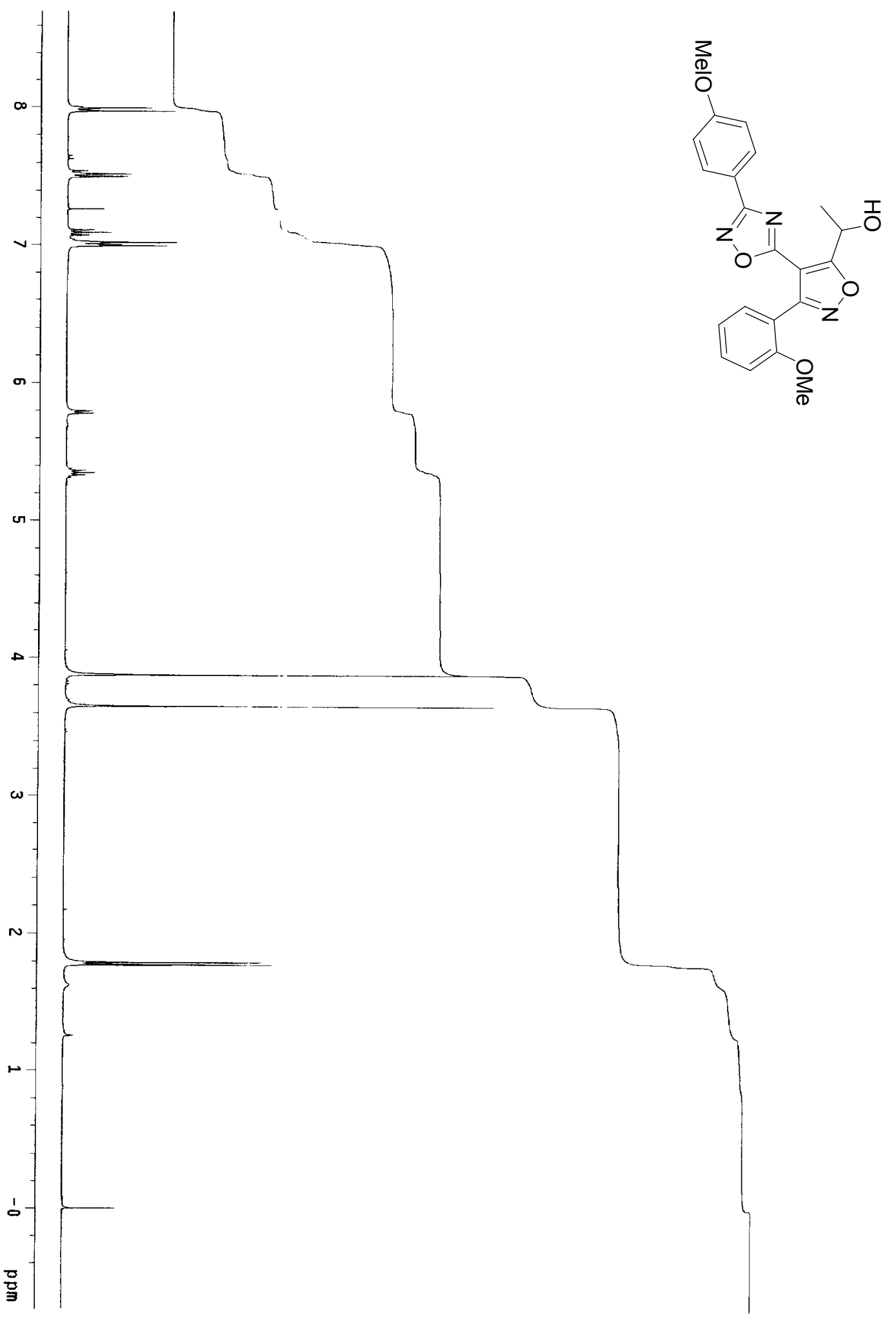



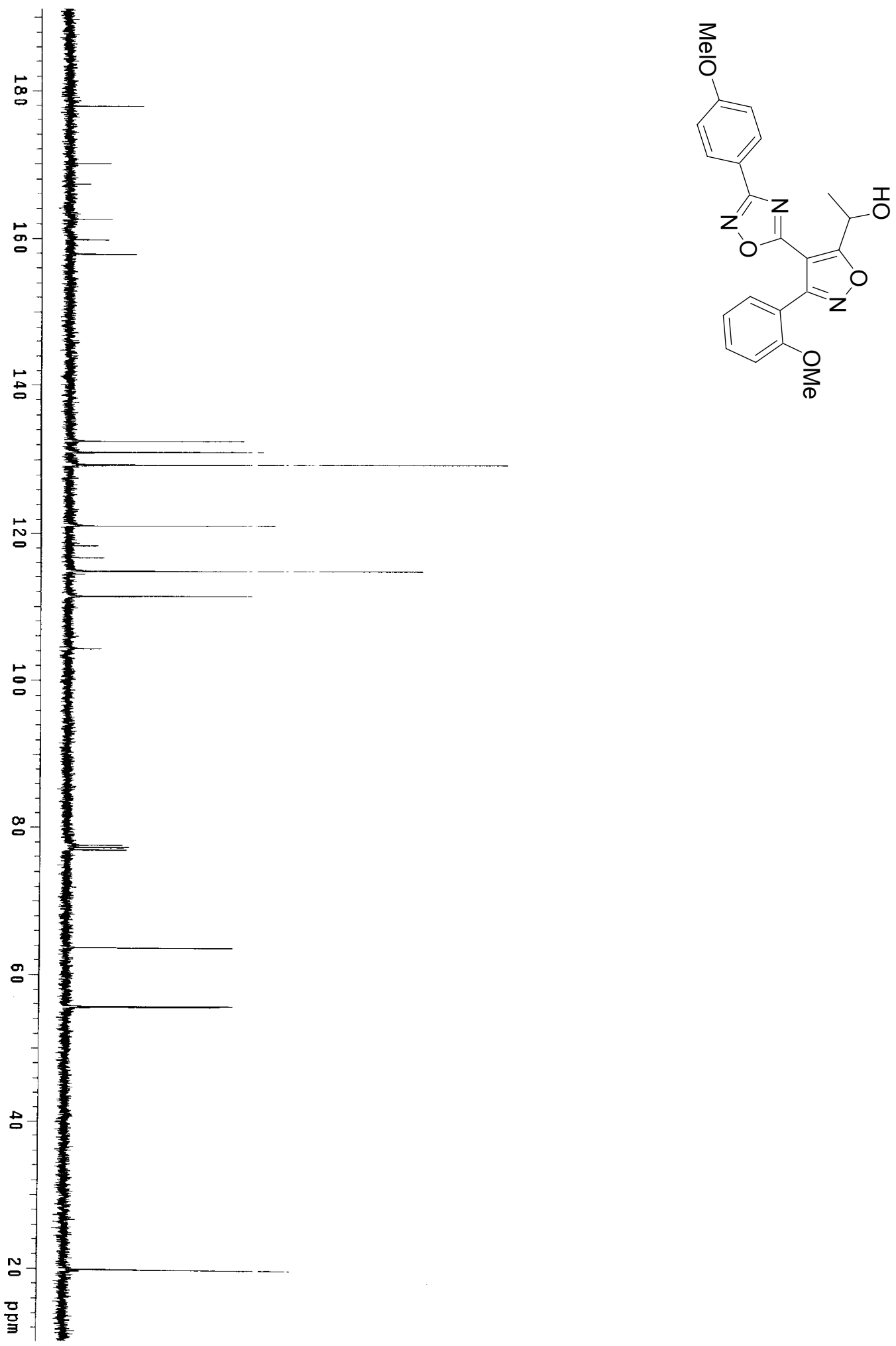


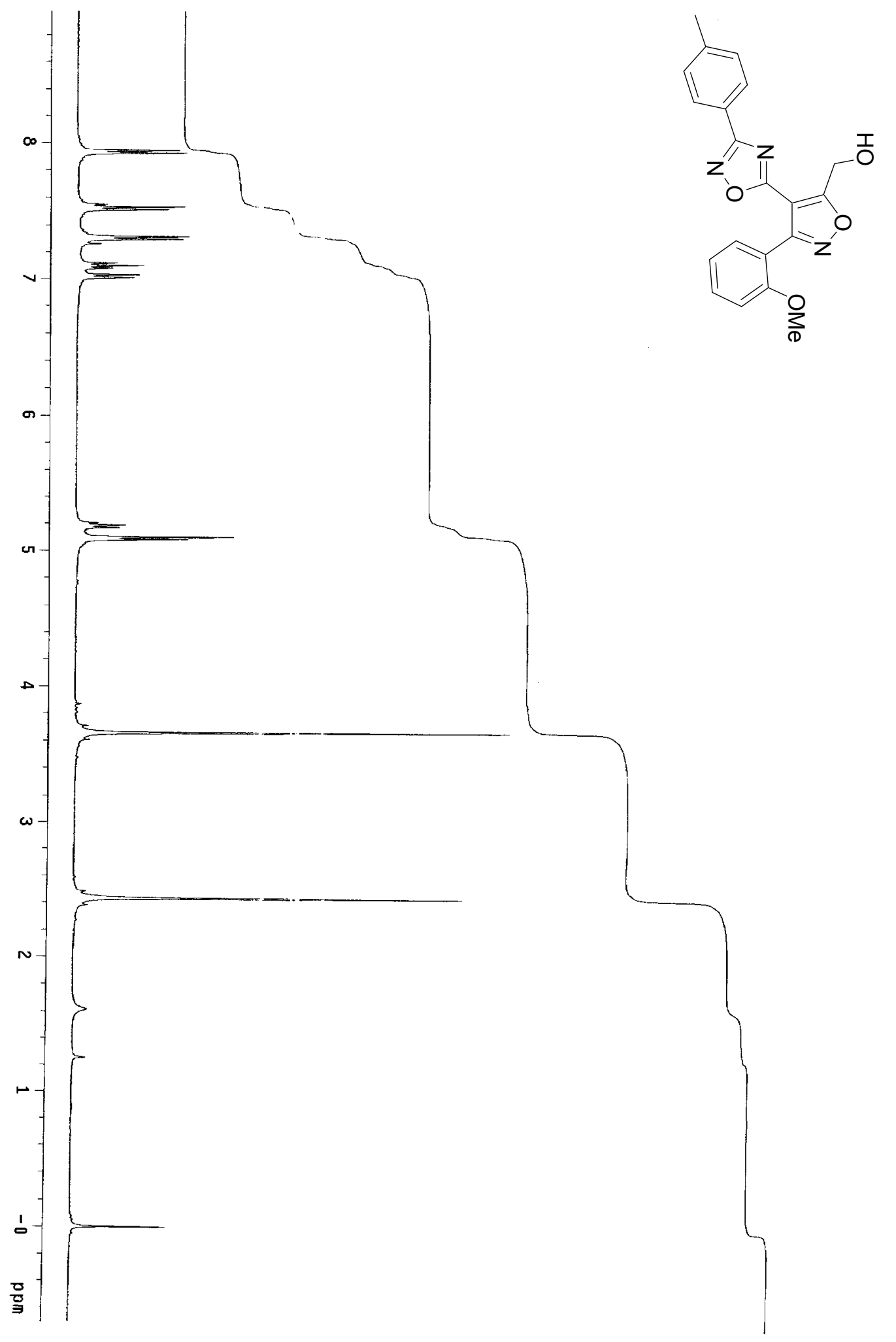



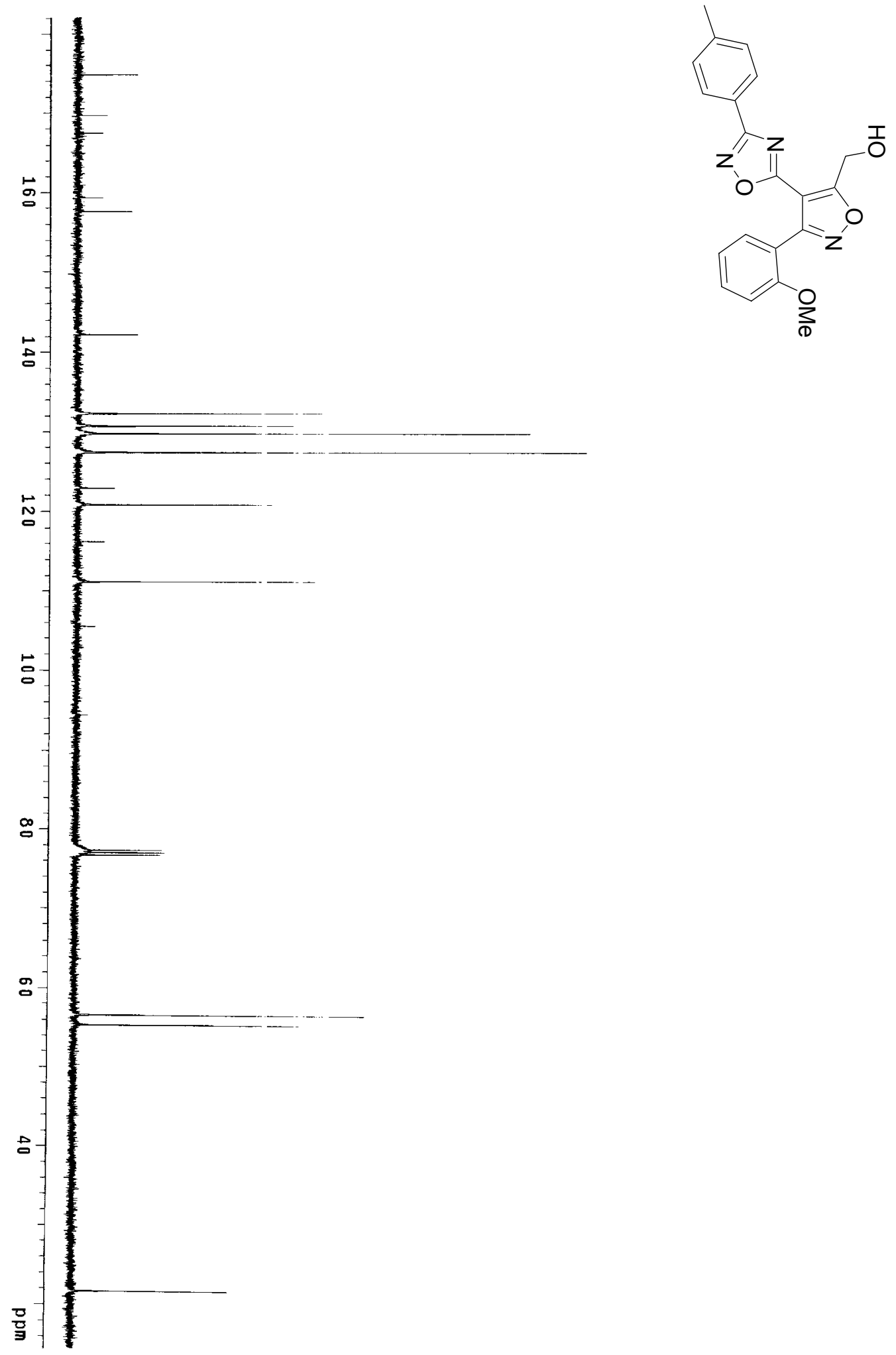


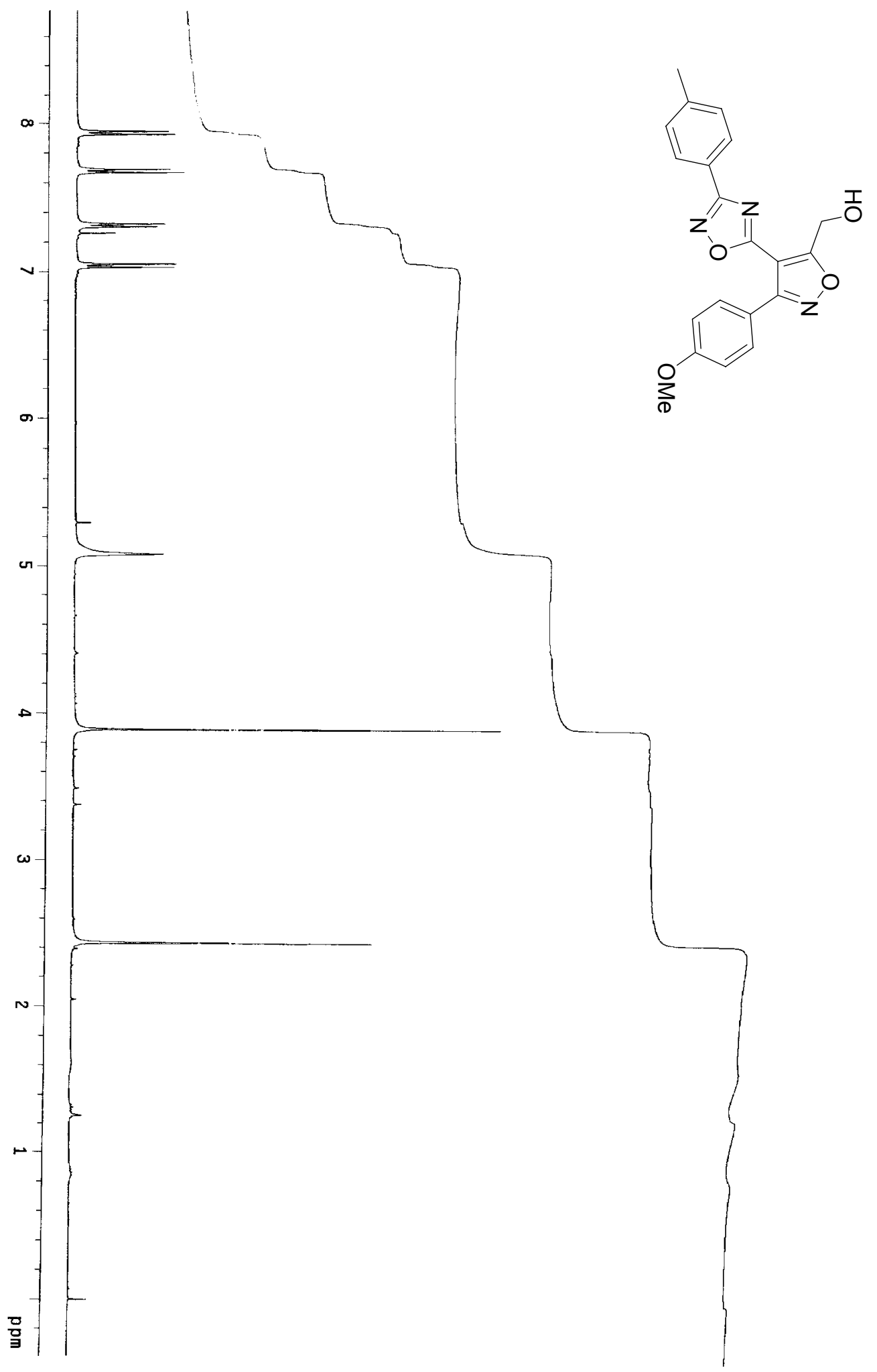



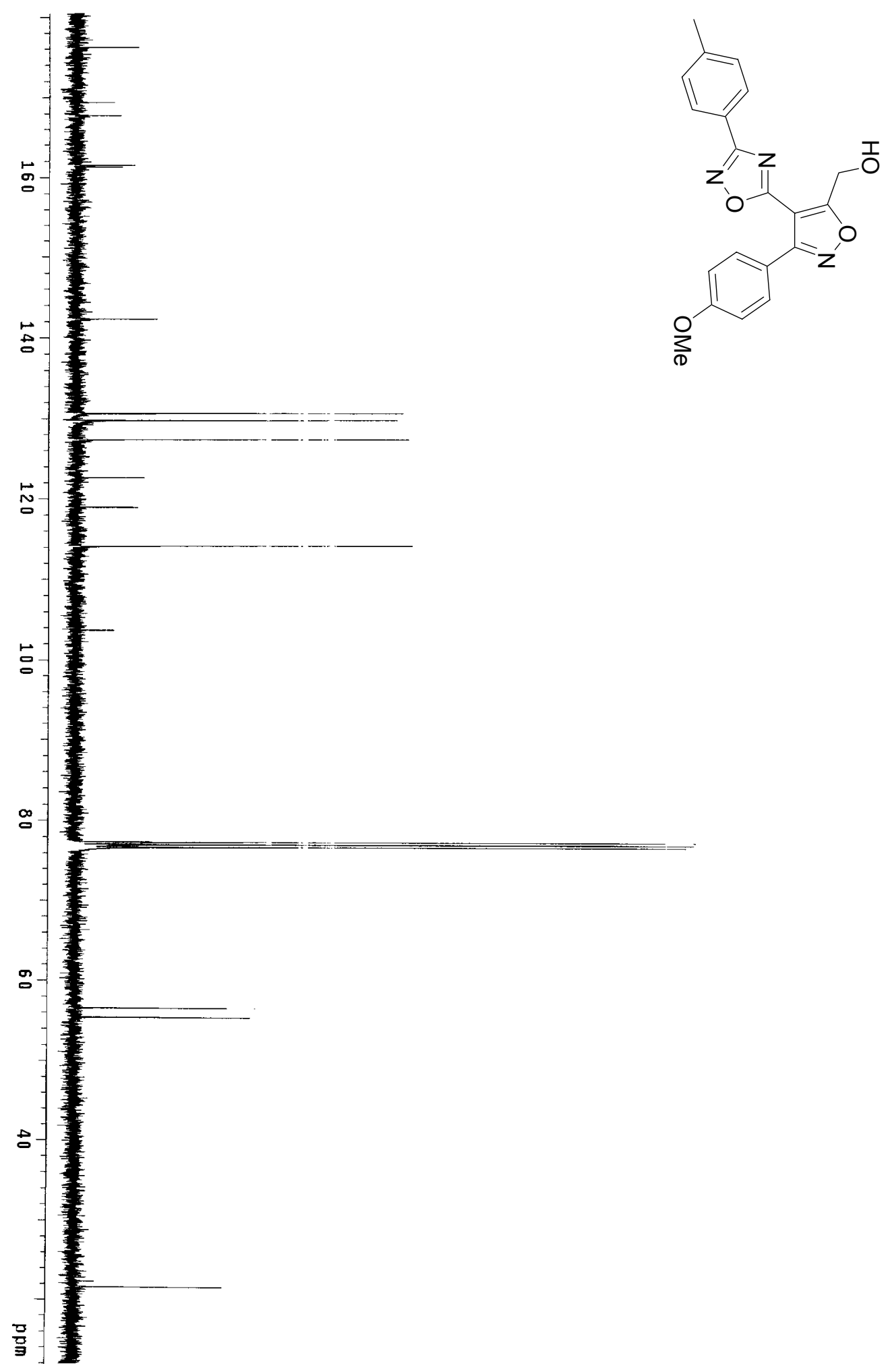


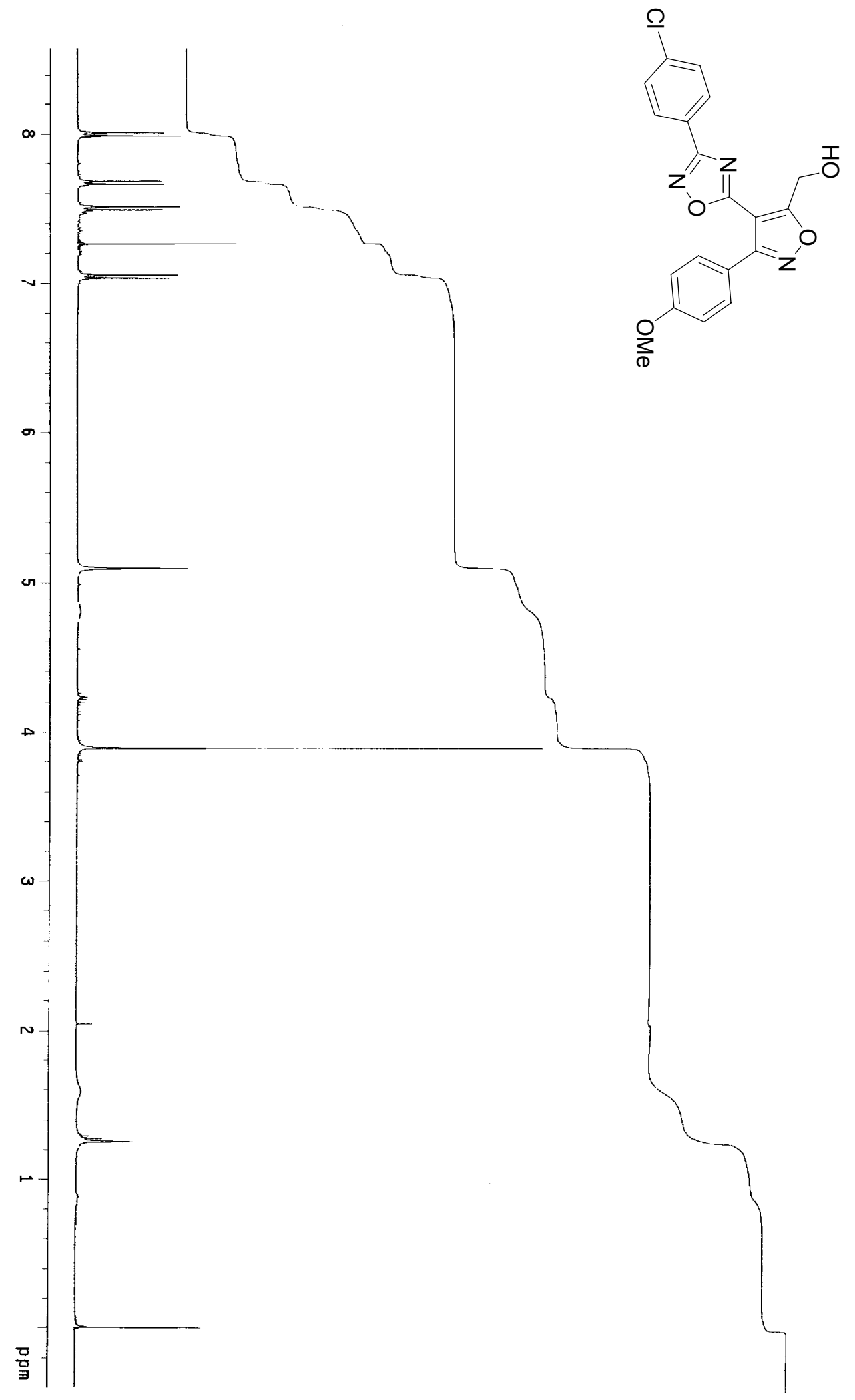



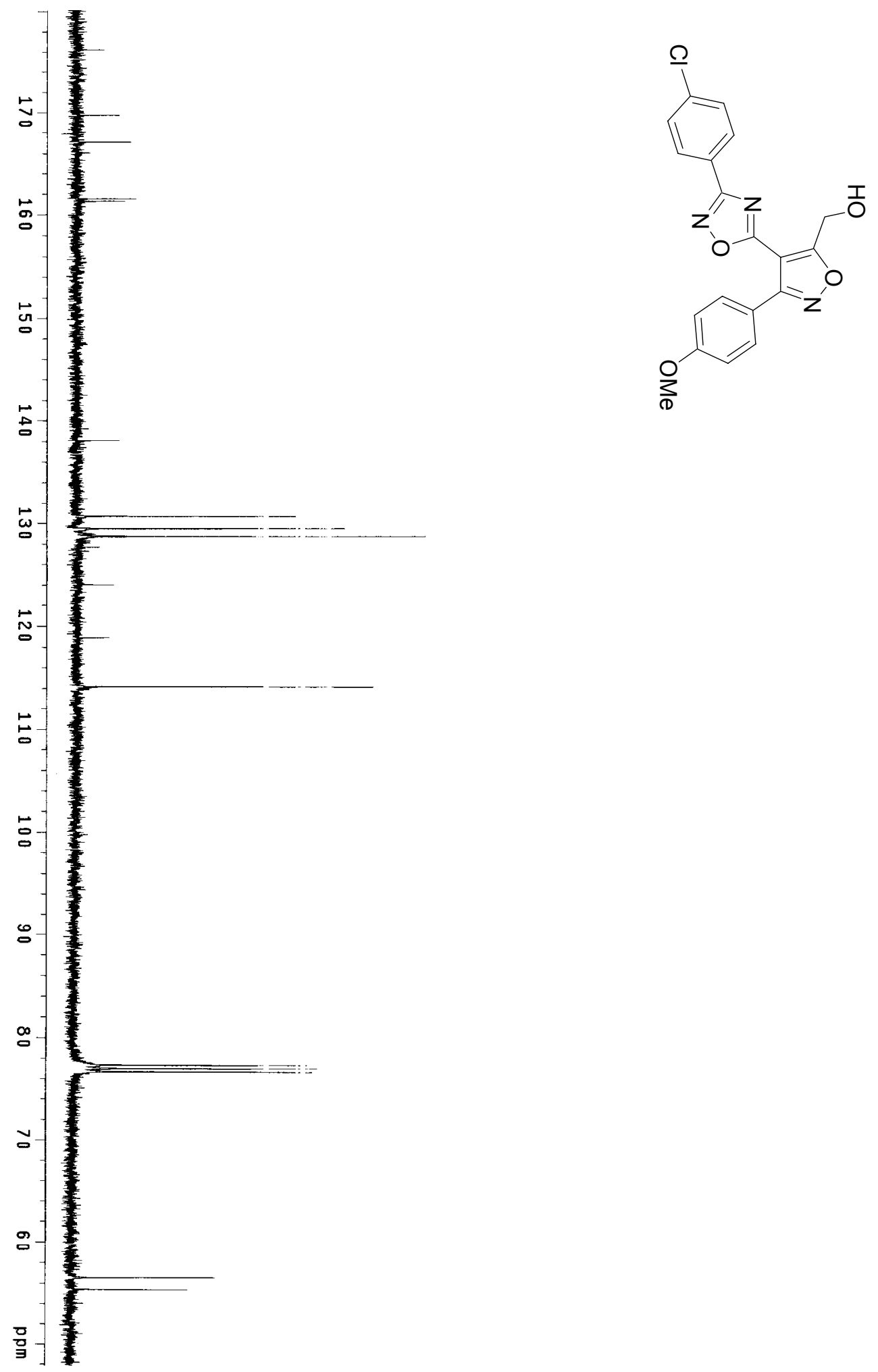


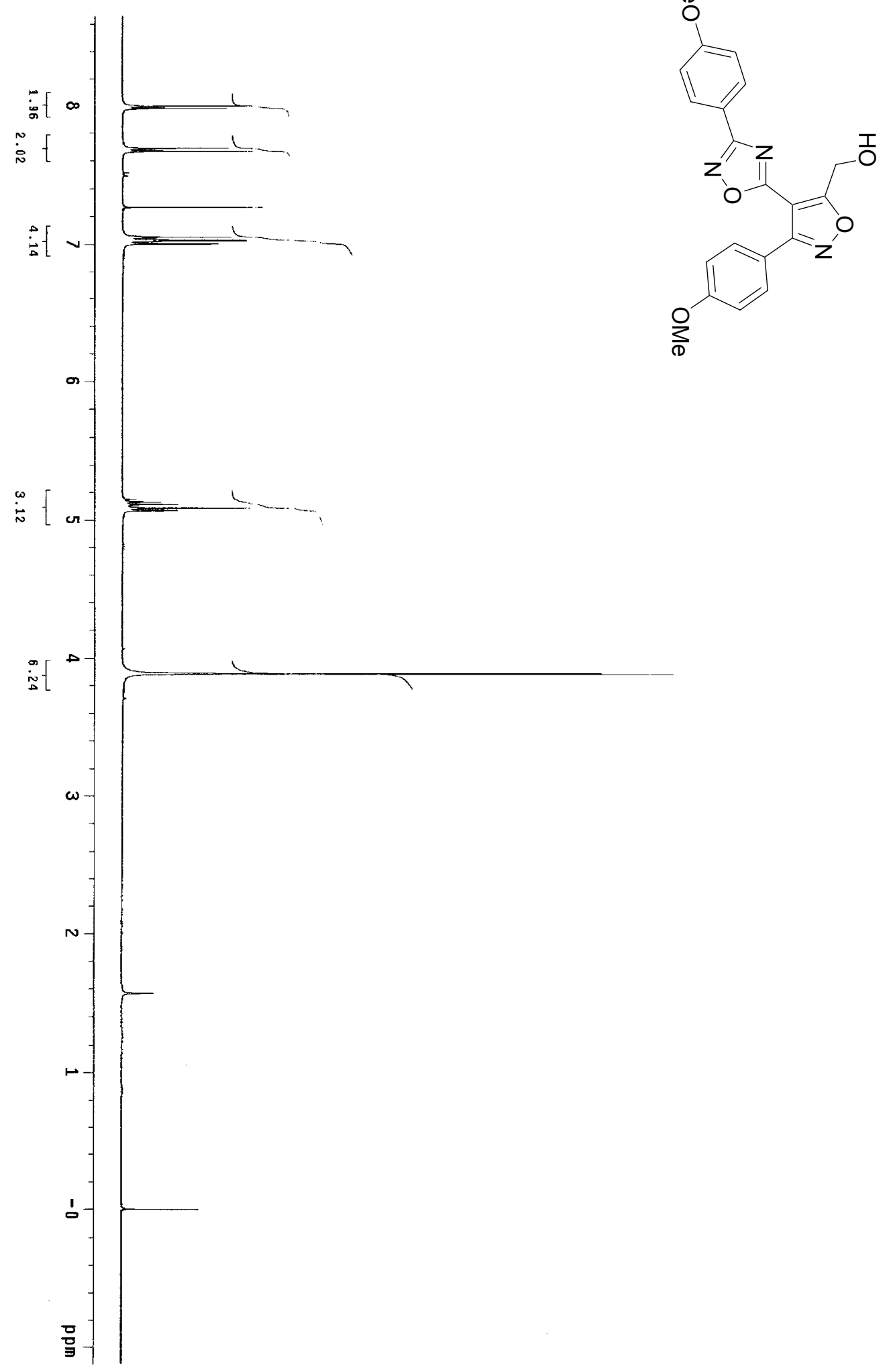




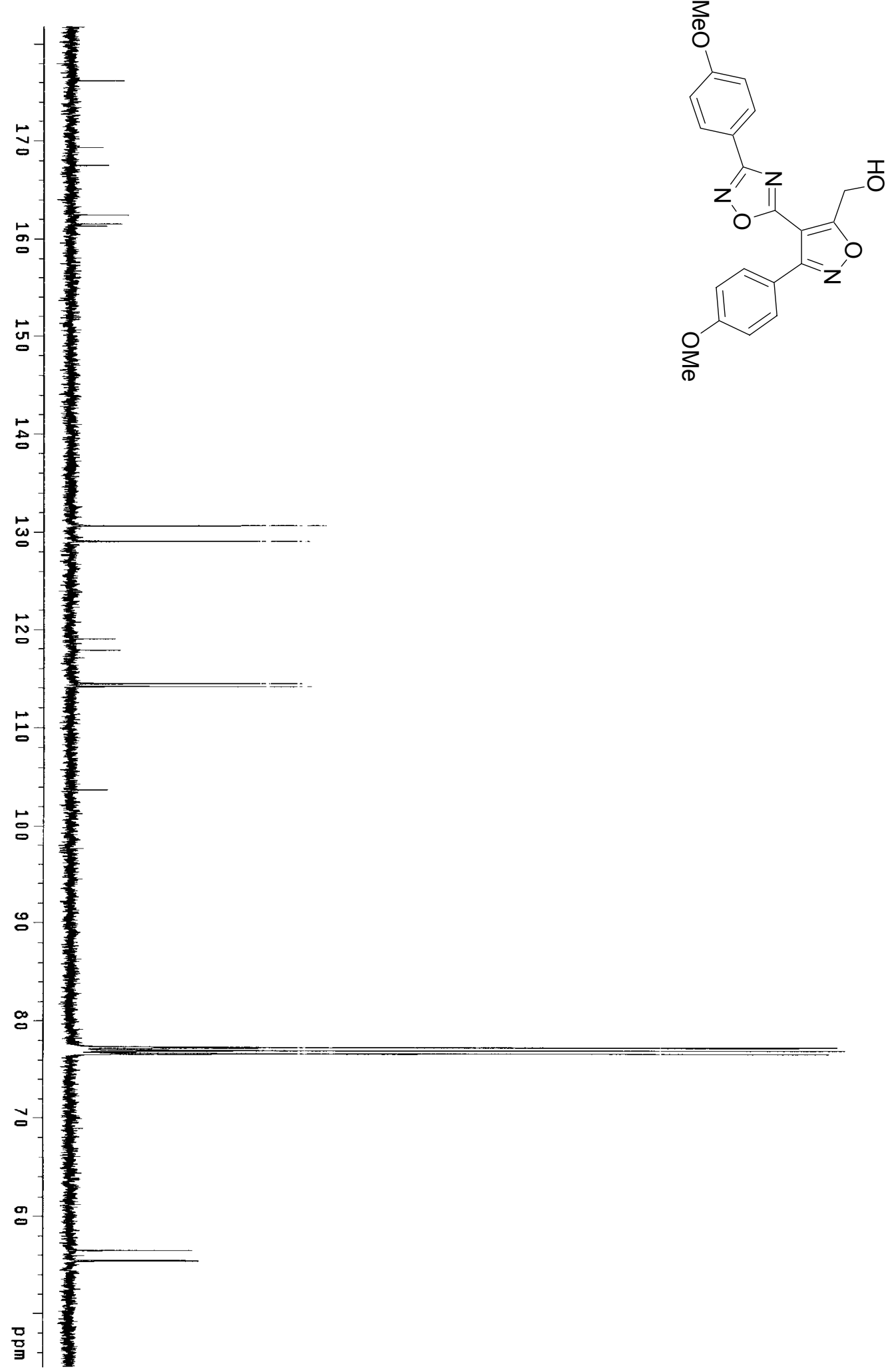

